\title{
A Case of Idiopathic Peripheral Neuropathy Related to a Running Injury Treated Successfully with Acupuncture
}

\author{
Yi-Chuan $\mathrm{C}^{1,2}$, Chun-Chang W1, Chih-Shan $\mathrm{H}^{3}$, Tsung-Jung $\mathrm{H}^{1,4,5,6}$, Yu-Chen $\mathrm{L}^{2,7}$ and Ing-Shiow $\mathrm{L}^{1,4,8 *}$ \\ ${ }^{1}$ Department of Chinese Medicine, China Medical University Beigang Hospital, Yunlin, Taiwan \\ ${ }^{2}$ Graduate Institute of Acupuncture Science, China Medical University, Taichung, Taiwan \\ ${ }^{3}$ Division of Neurology, China Medical University Beigang Hospital, Yunlin, Taiwan \\ ${ }^{4}$ Division of Chinese Medicine, Tainan Municipal An-Nan Hospital, Tainan, Taiwan \\ ${ }^{5}$ School of Sports Medicine, China Medical University, Taichung, Taiwan \\ ${ }^{6}$ School of Chinese Medicine, China Medical University, Taichung, Taiwan \\ ${ }^{7}$ Acupuncture department, China Medical University Hospital, Taichung, Taiwan \\ ${ }^{8}$ School of Post-baccalaureate Chinese Medicine, China Medical University, Taichung, Taiwan
}

\begin{abstract}
Idiopathic peripheral neuropathy lacks a specific cause. The risk of developing this condition increases with age and can seriously affect a patient's quality of life. Symptoms relate to whichever nerves are damaged and may progress slowly or remain unchanged after onset. No cure exists. Notably, acupuncture has proven efficacy in nerve regeneration and nerve function improvement. This case presentation describes a 60 -year-old male regular runner who developed right-sided drop foot after completing a marathon in June 2016. He was subsequently diagnosed as having a deep fibular nerve injury. After two months of physical rehabilitation, the right ankle remained dropped, with accompanying weakness and tightness in the anterior part of the right leg. In August 2016, the man commenced four months of electroacupuncture, three times a week. He experienced an obvious improvement in the velocity of motor nerve transduction and in F-wave testing. Moreover, there were marked improvements for the right ankle dorsiflexion range of motion in the supine $\left(120^{\circ}\right.$ to $\left.100^{\circ}\right)$ and seated $\left(15^{\circ}\right.$ to $\left.30^{\circ}\right)$ positions. The man resumed jogging in March 2017. Acupuncture may facilitate functional recovery after peripheral nerve injury.
\end{abstract}

Keywords: Deep fibular nerve; Running-related injury; Acupuncture

\section{Introduction}

Over 100 types of neuropathy have been identified, each of which has a different diagnosis. In peripheral neuropathy, damaged peripheral nerves transmit abnormal messages between the central nervous system (the brain and spinal cord) and muscles, organs, and other body tissues [1]. Causes of peripheral neuropathy vary; most commonly, neuropathies arise from a local cause such as nerve trauma, compression, or entrapment, from inflammatory processes in diseases such as diabetes mellitus, or from nerve damage such as in HIV infection. Although diabetes is a common cause of peripheral neuropathy, affecting approximately $30 \%$ of cases worldwide [2], in major studies, between $6 \%$ and $74 \%$ of neuropathy cases are labeled idiopathic [3]

The most common nerve problem affecting the lower extremities is thought to be lumbosacral radiculopathy, which leads to low back pain or sciatica symptoms [4]. Fibular neuropathy, the most frequent mononeuropathy in the lower limb and the third most common focal mononeuropathy after median and ulnar neuropathies [5], may lead to paresthesia, numbness, muscle weakness, and even drop foot.

Our patient suffered from drop foot after a routine 7-km run; his main complaint was associated with common fibular nerve injury. The most frequent site of injury to the nerve is just below the knee, where the nerve wraps around the lateral aspect of the fibula, immediately before dividing into deep and superficial branches. The deep branch, otherwise known as the deep fibular nerve (DFN), dominates ankle dorsiflexion and the sensory feeling of a web space between the first and second digits; the superficial branch, or superficial fibular nerve (SFN), dominates ankle eversion and the front and lateral feeling of the leg. Compression at the level of the fibular neck is frequently produced by external pressure on the nerve due to prolonged lying, crossing the legs, protracted squatting, and leg casts involving this site. Injury to the fibular head may severely affect the DFN [6].
The growing popularity of marathons in Taiwan in recent years has led to increasing numbers of people presenting to the clinic with running-related injuries (RRI), such as iliotibial band syndrome. Although some RRI are traumatic, most are due to overuse $[7,8]$. Generally, RRI is considered to be a musculoskeletal problem that is attributed to running, causing a restriction in running speed, distance, duration, or frequency, for at least 1 week. According to epidemiological studies, the incidence of RRI ranges from $18.2 \%$ to $92.4 \%$, while the prevalence ranges from 6.8 to 59 injuries per 1,000 hours of training [9]. There are few reports of running-related drop foot.

Typically, patients with peroneal neuropathy at the fibular neck present with acute foot drop, weakness of ankle dorsiflexion and they walk with a limp. They may also complain of paresthesia's and/ or sensory loss over the dorsum of the foot and lateral side, especially when the superficial branch is involved. In a cohort of 103 patients with common peroneal mononeuropathies, 79\% complained of sensory loss, but pain was relatively infrequent (16.5\%) [10].

In physical examination, fibular nerve neuropathy manifests as weakness in foot dorsiflexion and/or foot eversion, with normal inversion and plantar flexion. Sensory disturbance is confined to the dorsum of the foot, including the web space between the first and second digits and the lateral side. Nerve conduction studies (NCS) and electromyography (EMG) are very useful for confirming a diagnosis

*Corresponding author: Ing-Shiow L, Department of Chinese Medicine, China Medical University Beigang Hospital, Yunlin, Taiwan, Tel: +8865-7837901\#1229; E-mail: d5715@mail.bh.cmu.edu.tw

Received June 09, 2018; Accepted July 05, 2018; Published July 10, 2018

Citation: Yi-Chuan C, Chun-Chang W, Chih-Shan H, Tsung-Jung H, Yu-Chen L, et al. (2018) A Case of Idiopathic Peripheral Neuropathy Related to a Running Injury Treated Successfully with Acupuncture. J Clin Case Rep 8: 1140. doi: 10.4172/21657920.10001140

Copyright: (2018 Yi-Chuan C, et al. This is an open-access article distributed under the terms of the Creative Commons Attribution License, which permits unrestricted use, distribution, and reproduction in any medium, provided the original author and source are credited. 
Citation: Yi-Chuan C, Chun-Chang W, Chih-Shan H, Tsung-Jung H, Yu-Chen L, et al. (2018) A Case of Idiopathic Peripheral Neuropathy Related to a Running Injury Treated Successfully with Acupuncture. J Clin Case Rep 8: 1140. doi: 10.4172/2165-7920.10001140

Page 2 of 4

of fibular neuropathy. Such studies may identify conduction block at the site and it is possible to quantify the reduction in distal motor and sensory response amplitudes. Moreover, needle examination can determine nerve conduction in the DFN and SFN innervated muscles.

\section{Case Report}

A 60-year-old man, a regular runner, who denied having any chronic disease, including diabetes mellitus. In June 2016, after completing a marathon, he developed foot drop on the right side, despite no episodes of compression or falls during the day. $\mathrm{He}$ had no sensation of parenthesis or sensory problems in the lateral or anterior part of the right leg. NCS (Table 1) and EMG (Table 2) testing performed in a regional hospital indicated suspicion of rightsided DFN neuropathy with denervation signs below the knee. After two months of rehabilitation intervention, the right ankle remained dropped, with accompanying weakness and tightness in the anterior part of the right leg. In August 2016, after presenting to our department for consultation, the man received one 15-minute electroacupuncture (EA) session. According to his medical history and physical examination, the foot remained dropped both when walking and in the supine position. When we examined the extent of muscle power in the right ankle, focusing on the anterior tibialis, muscle power was fair with limited active dorsiflexion movement in the supine position, and unable to resist plantar flexion force. Whereas there was no limitation in the passive range of motion (PROM), the active range of motion (AROM) was limited when compared with the left side.

We selected a series of acupoints, including mainly ST36 (Zusanli), ST40 (Fenglung), GB34 (Yanglingchuan) and GB39 (Hsuanchung) for EA treatment, which was scheduled for two to three times a week at 2-3 Hz frequency (amplitude-modulated wave), for 15 minutes each session (Figure 1). The ST36 and ST40 acupoints are located near the DFN pathway; the GB34 and GB39 acupoints are situated close to the SFN pathway. We also selected the LR3 (Taichung) and ST41 (Jiexi) acupoints, for their proximity to the DFN pathway. During needle insertion, the patient sometimes complained of abnormal pain or a feeling of soreness compared with his previous experiences of acupuncture, and he felt a distinct numbness upon needle insertion at LR3.

After four months of regular EA treatment two to three times a week, improvements were noted in muscle power and the AROM. By

\begin{tabular}{|c|c|c|c|c|c|c|c|c|}
\hline \multicolumn{9}{|c|}{ Motor Summary Table } \\
\hline Right peroneal motor & \multicolumn{2}{|c|}{ Onset (ms) } & \multicolumn{2}{|c|}{ O-P Amp (mV) } & \multicolumn{2}{|c|}{ Dist. (cm) } & \multicolumn{2}{|c|}{$\operatorname{Vel}(\mathbf{m} / \mathbf{s})$} \\
\hline (EDB) & Ankle & Poplt & Ankle & Poplt & Ankle & Poplt & Ankle & Poplt \\
\hline $2016 / 06 / 30^{*}$ & NR & 12.7 & NR & 2.6 & $\mathrm{NR}$ & $\mathrm{NR}$ & \multicolumn{2}{|c|}{ NR } \\
\hline $21-02-2017$ & NR & NR & NR & NR & 8 & 32 & \multicolumn{2}{|c|}{ NR } \\
\hline $17-10-2017$ & 8.4 & 18.7 & 0 & 0.3 & 8 & 38 & \multicolumn{2}{|c|}{37} \\
\hline Left Peroneal Motor & \multicolumn{2}{|c|}{ Onset (ms) } & \multicolumn{2}{|c|}{ O-P Amp (mV) } & \multicolumn{2}{|c|}{ Dist. (cm) } & \multicolumn{2}{|c|}{$\operatorname{Vel}(\mathbf{m} / \mathbf{s})$} \\
\hline (EDB) & Ankle & Poplt & Ankle & Poplt & Ankle & Poplt & Ankle & Poplt \\
\hline $2016 / 06 / 30^{*}$ & 3 & 10.7 & 6.3 & 5.9 & -- & -- & \multicolumn{2}{|c|}{69} \\
\hline $21-02-2017$ & 4.1 & 11.7 & 3.9 & 3.7 & 8 & 34 & \multicolumn{2}{|c|}{45} \\
\hline $17-10-2017$ & 3.4 & 11.7 & 2.9 & 2.5 & 8 & 35 & \multicolumn{2}{|c|}{42} \\
\hline \multicolumn{9}{|c|}{ Anti-Sensory Summary Table } \\
\hline Right Sural Anti-Sensory & Onset & Peak & O-P Amp & \multirow{2}{*}{\multicolumn{2}{|c|}{ Site $A$ to $B$}} & Delta-0 & \multirow{2}{*}{ Dist. (cm) } & \multirow{2}{*}{$\operatorname{Vel}(\mathrm{m} / \mathrm{s})$} \\
\hline (Lat Mall) & (ms) & (ms) & (uV) & & & (ms) & & \\
\hline $2016 / 06 / 30^{*}$ & -- & 3.2 & -- & \multicolumn{2}{|c|}{ Leg to Ankle } & 2.5 & 12 & 48 \\
\hline $21-02-2017$ & 3.2 & 4.3 & 7.2 & \multicolumn{2}{|c|}{ Calf to Lat Mall } & 3.2 & 14 & 44 \\
\hline $17-10-2017$ & 3.1 & 3.9 & 7.2 & \multicolumn{2}{|c|}{ Calf to Lat Mall } & 3.1 & 14 & 45 \\
\hline Left Sural & Onset & Peak & O-P Amp & \multirow{3}{*}{\multicolumn{2}{|c|}{ Site $A$ to $B$}} & Delta-0 & & \\
\hline Anti-Sensory & \multirow{2}{*}{ (ms) } & \multirow{2}{*}{ (ms) } & \multirow{2}{*}{$(\mathrm{uV})$} & & & \multirow{2}{*}{ (ms) } & Dist. (cm) & $\operatorname{Vel}(\mathrm{m} / \mathrm{s})$ \\
\hline (Lat Mall) & & & & & & & & \\
\hline $21-02-2017$ & 3.2 & 4.3 & 8.7 & Calf & & 3.3 & 14 & 42 \\
\hline $17-10-2017$ & 2.9 & 4 & 10.7 & Calf & & 2.9 & 14 & 48 \\
\hline & & & Heflex St & & & & & \\
\hline Tibial & & & & & & 10 & (t) & \\
\hline (Gastroc) & & & & & & $L-R H-$ & (ms) & \\
\hline $2016 / 06 / 30^{*}$ & & & & & & & & \\
\hline $21-02-2017$ & & & & & & & & \\
\hline $17-10-2017$ & & & & & & & & \\
\hline & & & F Wave St & & & & & \\
\hline Peroneal & & & & & & $P F$ & (mc) & \\
\hline (Mrkrs)(EDB) & & & & & & L-k & (ms) & \\
\hline $2016 / 06 / 30^{*}$ & & & & & & & & \\
\hline $21-02-2017$ & & & & & & & & \\
\hline $17-10-2017$ & & & & & & & & \\
\hline $\begin{array}{l}\text { Data relating to June } 30,201 \\
\text { EDB: Extensor Digitorum Bre } \\
\text { Tibial: Tibial Nerve; Gastroc: } \\
\text { Latency; F-Lat: F Wave Laten }\end{array}$ & ocnemi & $\begin{array}{l}\text { tient wh } \\
\text { eak Amp } \\
\text { ll: Peror }\end{array}$ & $\begin{array}{l}\text { ed in a regi } \\
\text { t: Distance } \\
\text {; Mrkrs: M }\end{array}$ & $\begin{array}{l}\text { al. } \\
\text { tyy; Pop } \\
\text { Carget }\end{array}$ & $\begin{array}{l}\text { al Regio } \\
\text { t-Lat: H }\end{array}$ & $\begin{array}{l}\text { No Respo } \\
\text { Latency; }\end{array}$ & $\begin{array}{l}\text { Lat Mall: L } \\
\text { H-Lat: Left }\end{array}$ & al Malleolus \\
\hline
\end{tabular}

Table 1: Results of nerve conduction studies from different time points. 
Citation: Yi-Chuan C, Chun-Chang W, Chih-Shan H, Tsung-Jung H, Yu-Chen L, et al. (2018) A Case of Idiopathic Peripheral Neuropathy Related to a Running Injury Treated Successfully with Acupuncture. J Clin Case Rep 8: 1140. doi: 10.4172/2165-7920.10001140

Page 3 of 4

\begin{tabular}{|c|c|c|c|c|c|c|c|}
\hline Anterior Tibialis- & Ins Act & Fibs & Psw & Amp. & Dur. & Poly \\
\cline { 1 - 5 } DFN & None & $2+$ & $2+$ & None & None & None & None \\
\hline $2016 / 06 / 30^{*}$ & $\mathrm{Nml}$ & $\mathrm{Nml}$ & $\mathrm{Nml}$ & $\mathrm{Nml}$ & $\mathrm{Nml}$ & 0 \\
\hline $17-02-2017$ & $\mathrm{Nml}$ & $\mathrm{Nml}$ & $\mathrm{Nml}$ & $\mathrm{Nml}$ & $\mathrm{Nml}$ & $\mathrm{Nml}$ \\
\hline
\end{tabular}

*Data relating to June 30, 2016 was supplied by the patient who was tested in a regional hospital.

DFN: Deep Branch of Fibular Nerve; Ins Act: Insertional Activity; Fibs: Fibrillations; Psw: Positive Sharp Wave; Amp.: Amplitude; Dur.: Duration; Poly: Polyphasic Potentials; Recrt: Recruitment; Int Pat: Interference Pattern; Nml: Normal

Table 2: Electromyography records from different time points.

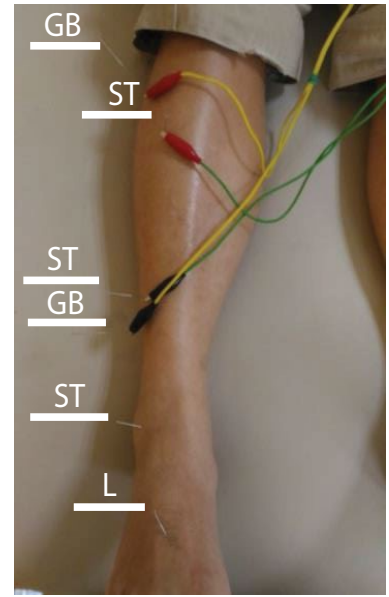

Figure 1: EA therapy for DFN and SFN.

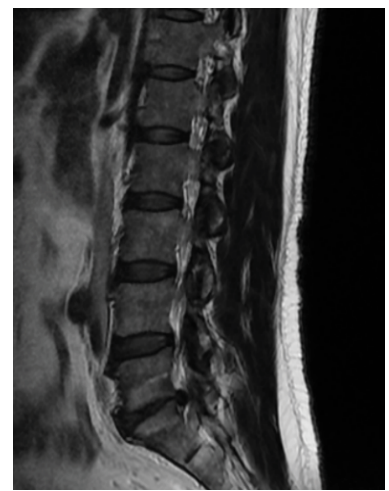

Figure 2: MRI image of the lumbar sacral region.

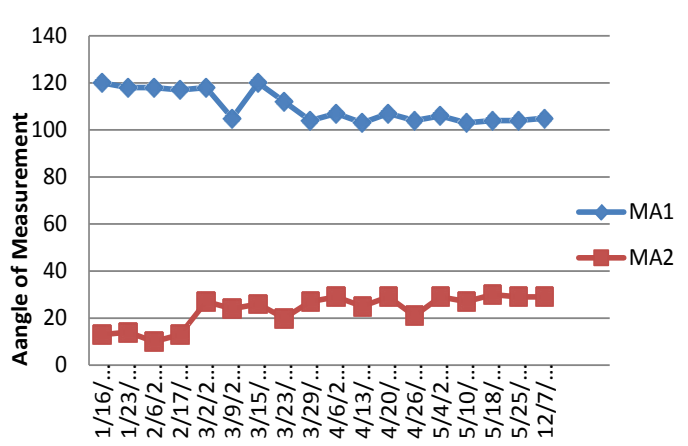

Figure 3: MA (measurement angle) 1 represents the angle of dorsiflexion between the tibia bone and the first metatarsophalangeal joint with the patient in the supine position. MA2 represents the angle of dorsiflexion between the fifth metatarsophalangeal joint and horizontal line with the patient in the seated position.
November 2016, the feeling of tightness had disappeared in the anterior part of the leg. Then, we asked the patient to undertake repeat NCS and EMG testing in our hospital's neurological division to evaluate nerve damage/recovery (Tables 1 and 2). The neurologist also arranged for an MRI scan to rule out any tumor mass in the spinal cord region. The MRI report was negative, but a degenerative disc disease was found with a slightly prominent hard disc at L5/S1 in the right side with neural foraminal stenosis and nerve root compression (Figure 2).

In March 2017, the man commented that the functional performance of his right leg was almost normal and he resumed jogging, so we decided to stop the treatment in May. At five months' follow-up (October 2017), we consulted the neurologist and conducted NCS and EMG testing to determine the improvement in nerve injury (Tables 1 and 2). During the EA intervention, we recorded AROM values from January through May 2017 (Figure 3). The angle of dorsiflexion between the tibia bone and the first metatarsophalangeal joint in the supine position (MA1; measurement angle 1) improved from $120^{\circ}$ to $100^{\circ}$, and the angle of dorsiflexion between the fifth metatarsophalangeal joint and horizontal line in the seated position (MA2; measurement angle 2) also improved from $15^{\circ}$ to $30^{\circ}$. In December 2017, even though EA had been discontinued for more than 6 months, there were clear improvements in motor nerve velocity and F-wave testing. In addition, EMG results of the DFN were at near-normal levels (Tables 1 and 2), AROM values were maintained (Figure 3 ) and the man was running as he had before the injury, twice a week for $7 \mathrm{~km}$ each time, without any discomfort.

\section{Discussion}

The classic treatment for fibular neuropathy involves removing the cause of compression or providing protection at the fibular neck, such as extra cushioning while sleeping and avoiding sitting with legs crossed during the day. In some cases, it can be useful to maintain the foot in a dorsiflexion position with an ankle-foot orthosis splint, which provides support and temporarily lessens the tension in the nerve and muscle until the injury has recovered. Physical therapy intervention is appropriate for rehabilitation. Based on nerve plasticity and the severity of the case, supervised training programs may lead patients step by step from PROM to passive-assistive, active, and active-resistant exercise. Walking is also an important therapy. According to each case, the therapist may evaluate performance and modulate rehabilitation programs to challenge the patient's ability to reach maximum potential for recovery.

In some refractory cases, operative decompression can be considered, especially when despite conservative treatment, symptoms and signs progress aggressively and no other cause is identified. Fortunately, in cases diagnosed with an entrapped nerve, surgical decompression is usually successful [11].

Acupuncture, a component of traditional Chinese medicine (TCM), is a well-known complementary and alternative treatment that has been used for thousands of years to treat diseases [12]. Evidence 
Citation: Yi-Chuan C, Chun-Chang W, Chih-Shan H, Tsung-Jung H, Yu-Chen L, et al. (2018) A Case of Idiopathic Peripheral Neuropathy Related to a Running Injury Treated Successfully with Acupuncture. J Clin Case Rep 8: 1140. doi: 10.4172/2165-7920.10001140

indicates that acupuncture can improve nerve activation, induce nerve repair, and even affect gene expression [13-15].

EA is frequently used instead of manual acupuncture, to provide stable and sustained acupuncture stimulation. Much clinical evidence has shown that EA improves blood flow and induces vascularization in the local area of insertion [16] and can promote both motor and sensory neuron regeneration $[17,18]$. Furthermore, EA may not only improve the morphological performance of motor and sensory neurons in the process of nerve fiber regeneration, but also their functional performance [19].

According to the TCM system, the ST meridian course in the leg region is situated 1 can away from the anterior crest of the tibia, which extends from the space between the femoral and lateral condyle of the tibia, near the ST35 (Dubi) acupoint, to the ankle region between the extensor pollicis longus tendon and the extensor digitorum longus tendon, near the ST41 acupoint. We selected the ST36 and ST40 acupoints for EA treatment in order to stimulate the DFN and the anterior tibialis, a muscle that is principally involved in ankle dorsiflexion.

The sensory distribution of the DFN is felt over the web space between the first and second digits near the LR3 acupoint. Insertion of this acupoint for some sensory input of DFN may provide sensory feedback and promote nerve recovery. Conversely, the GB meridian course in the leg region runs from the lateral part along the anterior board of the fibula bone from the anterior-inferior portion of the fibular head, at the GB34 acupoint, to the anterior space of the lateral malleolus, at the GB40 (Chiuhsu) acupoint. Selecting GB34 and GB40 for EA treatment is mainly for the purpose of stimulating the SFN for feedback of sensory input.

Some research has indicated that the sensitivity of MRI is much lower than expected in the detection of lumbar nerve root compromise [20]. The findings from the MRI report for this case revealed neural foraminal stenosis and nerve root compression at L5/S1 on the right side, but the clinical manifestations and history of sudden-onset of drop foot were not consistent with nerve root compression, which is usually progressive and accompanied by a feeling of numbness in the lower extremity.

\section{Conclusion}

The meridian courses, or energy pathways, which according to TCM flow throughout the body, do not closely follow the nerve pathways identified by Western medicine. Yet, we lack satisfactory explanations for the observed efficacy of acupuncture intervention in a multitude of symptoms and syndromes. It appears in this case that the delivery of EA at acupoints near the DFN induced nerve recovery and improved functional performance. Further explorations are needed to determine the efficacy of EA in the treatment of peripheral neuropathy.

\section{References}

1. Torpy JM, Kincaid JL, Glass RM (2010) JAMA patient page. Peripheral neuropathy. JAMA 303: 1556.

2. Stino AM, Smith AG (2017) Peripheral neuropathy in prediabetes and the metabolic syndrome. J Diabetes Investig 8: 646-655.

3. Singe MA, Vernino SA, Wolfe GI (2012) Idiopathic neuropathy: New paradigms, new promise. J Peripher Nerv Syst 2: 43-49.

4. Tarulli AW, Raynor EM (2007) Lumbosacral radiculopathy. Neurol Clin 25: 387-405.

5. Marciniak C (2013) Fibular (peroneal) neuropathy: Electrodiagnostic features and clinical correlates. Phys Med Rehabil Clin N Am 24: 121-137.

6. Poage C, Roth C, Scott B (2016) Peroneal nerve palsy: Evaluation and management. J Am Acad Orthop Surg 24: 1-10.

7. Vitez L, Zupet $P$, Zadnik V, Drobnič $M(2017)$ Running injuries in the participants of ljubljana marathon. Zdr Varst 56: 196-202.

8. Hreljac A (2005) Etiology, prevention, and early intervention of overuse injuries in runners: A biomechanical perspective. Phys Med Rehabil Clin N Am 16: 651-667.

9. Saragiotto BT, Yamato TP,. Hespanhol LCJ, Rainbow MJ, Davis IS, et al. (2014) What are the main risk factors for running-related injuries? Sports Med 44: 1153-1163.

10. Katirji MB, Wilbourn AJ (1988) Common peroneal mononeuropathy: A clinical and electrophysiologic study of 116 lesions. Neurolo 38: 1723-1728.

11. Mont MA, Dellon AL, Chen F, Hungerford MW, Krackow KA, et al. (1996) The operative treatment of peroneal nerve palsy. J Bone Joint Surg Am 78: 863869.

12. Kaptchuk TJ (2002) Acupuncture: Theory, efficacy, and practice. Ann Intern Med 136: 374-383.

13. Fu SP, He SY, Xu B, Hu CJ, Lu SF, et al. (2014) Acupuncture promotes angiogenesis after myocardial ischemia through $\mathrm{H} 3 \mathrm{~K} 9$ acetylation regulation at VEGF gene. PLoS One 9: 94604

14. Li WJ, Li SM, Ding Y, He B, Keegan J, et al. (2012) Electro-acupuncture upregulates CGRP expression after rat spinal cord transection. Neurochem Int 61: 1397-1403.

15. Wang W, Li J, Meng X, Chen Y (2015) Effect of electronic stimulation at Neiguan (PC 6) acupoint on gene expression of adenosine triphosphate-sensitive potassium channel and protein kinases in rats with myocardial ischemia. J Tradit Chin Med 35: 577-582.

16. Chen YS, Yao $\mathrm{CH}$, Chen $\mathrm{TH}$, Lin JG, Hsieh CL, et al. (2001) Effect of acupuncture stimulation on peripheral nerve regeneration using silicone rubber chambers. Am J Chin Med 29: 377-385

17. Brushart TM, Hoffman PN, Royall RM, Murinson BB, Witzel C, et al. (2002) Electrical stimulation promotes motoneuron regeneration without increasing its speed or conditioning the neuron. J Neurosci 22: 6631-6638.

18. Elzinga K, Tyreman N, Ladak A, Savaryn B, Olson J, et al. (2015) Brief electrical stimulation improves nerve regeneration after delayed repair in Sprague Dawley rats. Exp Neurol 269: 142-153.

19. Hoang NS, Sar C, Valmier J, Sieso V, Scamps F (2012) Electro-acupuncture on functional peripheral nerve regeneration in mice: A behavioural study. BMC Complement. Altern Med 12: 141.

20. Tawa N, Rhoda A, Diener I (2016) Accuracy of magnetic resonance imaging in detecting lumbo-sacral nerve root compromise: A systematic literature review. BMC Musculoskelet Disord 17: 386. 